\title{
OPEN Probiotic Lactobacillus fermentum strain JDFM216 improves cognitive behavior and modulates immune response with gut microbiota
}

Mi Ri Park ${ }^{1,8}$, Minhye Shin ${ }^{2,8}$, Daye Mun ${ }^{2}$, Seong-Yeop Jeong ${ }^{3}$, Do-Youn Jeong ${ }^{3}$, Minho Song ${ }^{4}$ Gwangpyo $\mathrm{Ko}^{5}$, Tatsuya Unno ${ }^{5,6}$, Younghoon $\mathrm{Kim}^{2 \varpi}$ \& Sangnam $\mathrm{Oh}^{7 凶}$

Increasing evidence indicates that alterations in gut microbiota are associated with mammalian development and physiology. The gut microbiota has been proposed as an essential player in metabolic diseases including brain health. This study aimed to determine the impact of probiotics on degenerative changes in the gut microbiota and cognitive behavior. Assessment of various behavioral and physiological functions was performed using Y-maze tests, wheel running tests, accelerated rotarod tests, balance beam tests, and forced swimming tests (FSTs), using adult mice after $\mathbf{5 0}$ weeks of administering living probiotic bacterium Lactobacillus fermentum strain JDFM216 or a vehicle. Immunomodulatory function was investigated using immune organs, immune cells and immune molecules in the mice, and gut microbiota was also evaluated in their feces. Notably, the $L$. fermentum JDFM216-treated group showed significantly better performance in the behavior tests $(P<0.05)$ as well as improved phagocytic activity of macrophages, enhanced slgA production, and stimulated immune cells $(P<0.05)$. In aged mice, we observed decreases in species belonging to the Porphyromonadaceae family and the Lactobacillus genus when compared to young mice. While administering the supplementation of $L$. fermentum JDFM216 to aged mice did not shift the whole gut microbiota, the abundance of Lactobacillus species was significantly increased $(P<0.05)$. Our findings suggested that $L$. fermentum JDFM216 also provided beneficial effects on the regulation of immune responses, which has promising implications for functional foods. Taken together, L. fermentum JDFM216 could confer the benefit of improving health with enhanced cognition, physiological behavior, and immunity by modulating the gut microbiota.

The prevalence of aging-associated diseases has been increased according to the arising of elderly population, and functional foods that furnish health-promoting effects to control aging and longevity including health span have become more desirable. Physiological changes related with aging include impaired memorial and cognitive functions, suppressed immune responses, and dysbiosis of bone remodeling ${ }^{1}$. Importantly, environments in early life could remarkably influence on later development, maturation, and functionality of the host. Especially, gut microbiota co-exists in a mostly symbiotic partnership with its host during the entire life cycle. At birth, colonization by the microbiota participates in maturation of intestinal barrier function and gut homeostasis as well as immune response including innate and host adaptive immunity ${ }^{2}$. The microbiota undergoes dramatic changes in richness and composition during adulthood, contributing to cellular homeostasis and disease onset in later life ${ }^{3}$.

The dynamics of gut microbiota have been increasingly recognized to modulate host physiology and are linked to central nervous system function in human and animal studies ${ }^{4,5}$. Importantly, emerging evidences propose that a gut-brain axis exists and that it plays an important role in modulating cognitive functions and behavior ${ }^{6}$.

${ }^{1}$ Department of Animal Science and Institute of Milk Genomics, Jeonbuk National University, Jeonju 54896, South Korea. ${ }^{2}$ Department of Agricultural Biotechnology and Research Institute of Agriculture and Life Science, Seoul National University, Seoul 08826, South Korea. ${ }^{3}$ Microbial Institute for Fermentation Industry, Sunchang, Jeonbuk 56048, South Korea. ${ }^{4}$ Division of Animal and Dairy Science, Chungnam National University, Daejeon 34134, South Korea. ${ }^{5}$ Subtropical/Tropical Organism Gene Bank, Jeju National University, Jeju 63243, South Korea. ${ }^{6}$ Faculty of Biotechnology, Jeju National University, Jeju 63243, South Korea. ${ }^{7}$ Department of Functional Food and Biotechnology, Jeonju University, Jeonju 55069, South Korea. ${ }^{8}$ These authors contributed equally: Mi Ri Park and Minhye Shin. ${ }^{\square}$ email: ykeys2584@snu.ac.kr; osangnam@jj.ac.kr 
Many studies have shown the microbiota and its role in neurological disorders such as multiple sclerosis, autism, Parkinson's disease, and Alzheimer's disease ${ }^{7-11}$. And supplementation of probiotics is highly associated with neuroprotective effects in hosts with the brain disorders. Therefore, the modulation of gut microbiota may be new strategy for cognitive disorders related to neurodegenerative diseases and depression as targeting new pathway.

Probiotics, live microorganisms that provide health benefit to the host when administered in adequate amounts, reveal on strong interaction with gut commensals. Among them, it has been well-established that therapeutic interventions of lactobacilli improve cognitive behaviors for mitigating mood disorders as well as alters gut microbiota composition ${ }^{12-14}$. Moreover, supplementation with probiotics restores harmful effects observed on the immune and cognitive functions due to aging through anti-inflammation and anti-oxidative stress $^{15}$. Among the species of Lactobacillus, it has been reported that Lactobacillus fermentum confers symbiotic impacts by stimulation of effective immunity and antioxidant activity in aging mice ${ }^{16}$. Similarly, in our previous studies, we showed that one strain of Lactobacillus species, L. fermentum JDFM216, exerts probiotic properties such as adhesive properties, lifespan extension, strengthening of the immune system and other health-promoting functions in a Caenorhabditis elegans animal model ${ }^{17}$. Here we focused on understanding whether and how microbiota alterations by supplementation with L. fermentum JDFM216 contribute to behavior and immune responses, and whether physical and mental decline could be targeted by gut microbiota modulations using the aged mouse model.

\section{Results}

JDFM216 affected the behaviors of aged mouse. In the current study, our research objective was to understand how probiotic supplementation contributes to behavior and immune responses in the aged mouse model, since a number of human clinical trials have been reported on the effects of long-term probiotics supplementation in middle-aged and older adults to examine their impact on cognitive functions ${ }^{18-21}$. Therefore, we employed 12-month-old ( 52 weeks) mice as a model of middle-aged mice according to the relation between mice age and human age ${ }^{22}$, and JDFM216 was administered for 48 weeks to ensure that senescence was sufficiently developed.

This study first aimed to assess the effects of JDFM216 on age-related cognition, memory impairment and brain functions. In the Y-maze test, oral administration of JDFM216 significantly increased spontaneous alternation by $4.46 \%$ compared with the control group (Fig. 1B; $P=0.0288$ ). All aged mice with JDFM216 treatment significantly alternated between arms above chance level at $22.2 \%$, while the performance of the control group was not different from the level. Since a higher percentage of alternations has been associated with better recognition and thus increased memory, these data implicate that JDFM216 may improve spatial working memory in aged mice. It is noted that although the Y-maze test is not the most specific test for memory, Y-maze function is sensitive to damage in areas such as the hippocampus highly associated with cognition and neurodegenerative diseases ${ }^{23}$. Also, these data are consistent with prior observations that L. plantarum C29 strain ameliorates memory impairment caused by aging ${ }^{24}$, implying the probable efficacy of JDFM216 for improvement of memory. We next conducted the wheel-running test for voluntary exercises to determine locomotor activities. Spontaneous running activities were not significantly different between the control and JDFM216-treated groups, and mice ran an average of approximately 3.0 yards for $5 \mathrm{~min}$ in both groups (Fig. 1C). These findings indicate that dietary supplementation with JDFM216 has no effect on the capacity for voluntary exercise. Motor learning abilities were also measured by the accelerating rotarod test. This test is widely used to evaluate the motor coordination of rodents and is especially sensitive for detecting cerebellar dysfunction. In this test, motor performances of mice were analyzed by measuring the latency to falling off a rod that was rotated with increasing velocity. As a result, the JDFM216-treated group showed a significantly increased latency to fall by $88.6 \%$ compared with the control group (Fig. 1D; $P=0.0234$ ), suggesting that JDFM216 enhances motor performance in aged mice. Sense of balance and movement function of the back foot of the aged mice were further assessed using the balance beamwalking test, which analyses the number of foot slips. Mice were trained to traverse a medium ( $40 \mathrm{~mm}$ wide) square beam for 2 days, and the number of hind paw slips was scored and video-recorded on the test days on a narrow (30 mm wide) beam. As a result of the test, JDFM216-treated mice showed significantly higher scores with fewer slips by $47.9 \%$ compared with control mice (Fig. $1 \mathrm{E} ; \mathrm{P}=0.0046$ ), suggesting better motor balance ability. It is worth mentioning that mice are known to lose their visual acuity with age ${ }^{25}$. The effect of JDFM 216 on the improved motor balance could result from alleviated loss of the visual function in aged mice by the JDFM216 administration, and further investigation is required to specify the mechanism.

Currently, depression is one of the most common psychiatric disorders and has become a public health concern, since it is associated with high morbidity and mortality. Thus, we finally performed the forced swimming test (FST), which is the most widely used behavioral test to measure the level of depression of drugs and compounds. Notably, this test demonstrated that JDFM216 exhibited an antidepressant-like effect by showing a significantly decreased immobility time by $19.6 \%$ compared with the control group (Fig. $1 \mathrm{~F} ; P=0.0353$ ). We also weighed all the mice biweekly during the whole period of studies, and there were no significant differences in body weight gain between the groups that received the JDFM216 treatment and the control up to beginning the behavioral tests. Importantly, the mean percentage of weight loss changed significantly between the two groups before (at 100 weeks) and after (at 102 weeks) the behavioral assessments (Fig. 1G, P=0.0018). Aged mice in the control group lost, on average, approximately $10.8 \%$ of their body weight after the overall behavior tests, and those in the JDFM216-treated group showed a 3.1\% weight loss (approximately $7.4 \pm 0.2 \mathrm{~g}$ lighter in the control mice than those that received treatment). We speculate that the difference may be because mice in the control group were more vulnerable to stress. Altogether, these results indicate that the JDFM216 might have an effect in aged mice manifesting as resistance to environmental stresses from continuous test-related behavior, 
(A)

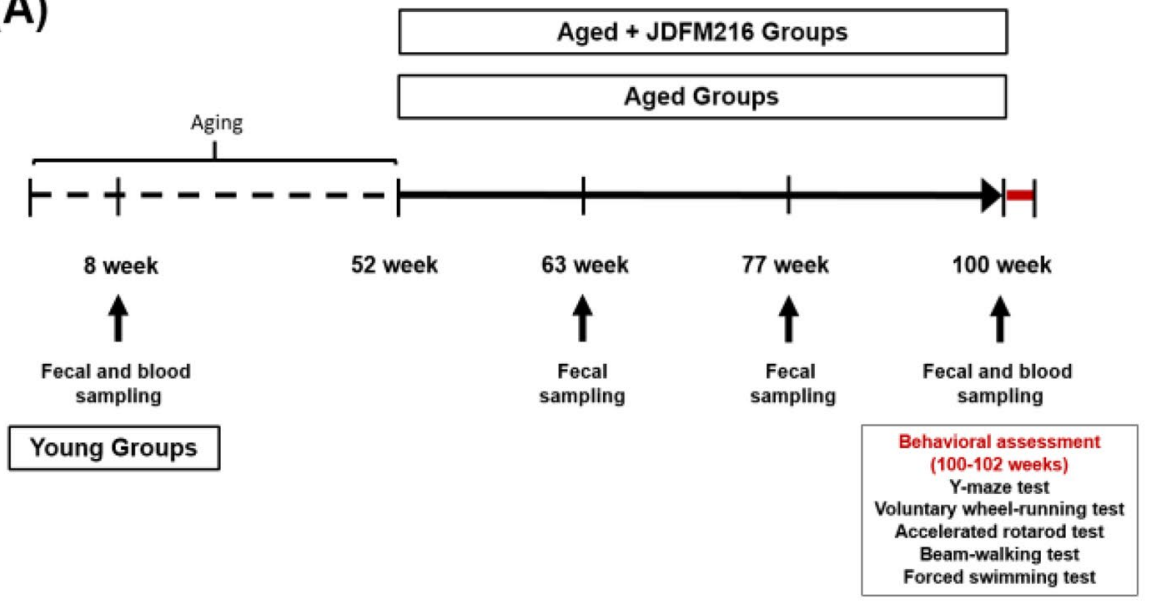

(B)

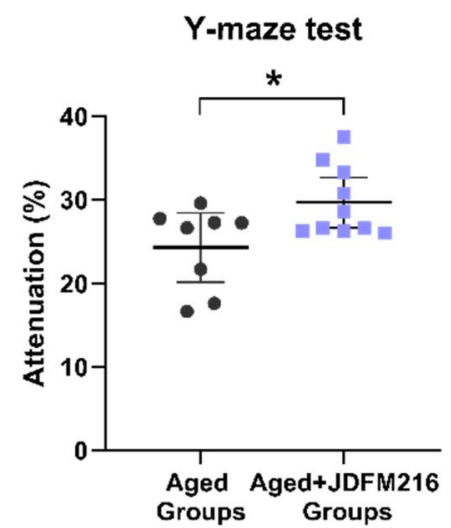

(E)

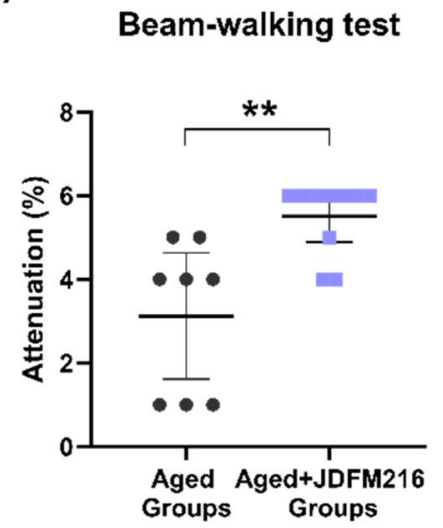

(C)
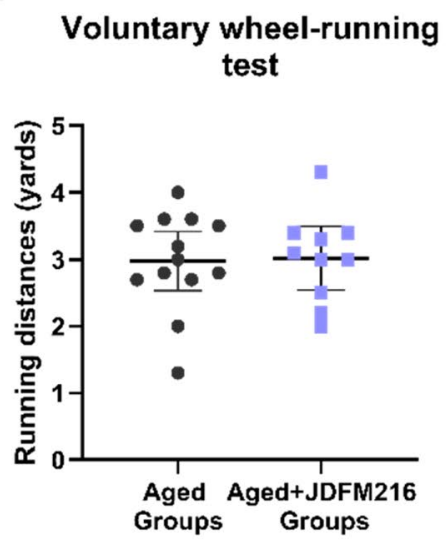

(F)

Forced swimming test

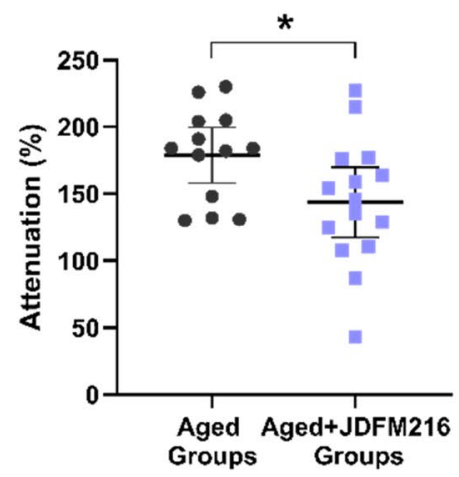

(D)

Accelerated rotarod test

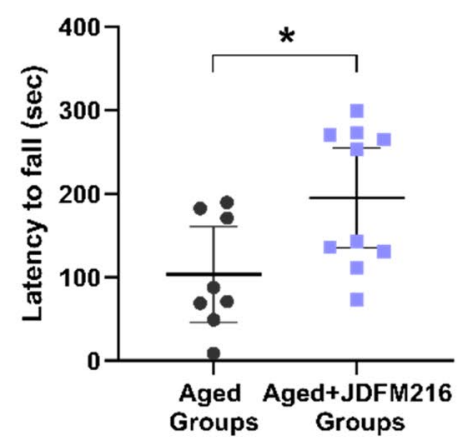

(G)

\section{Body weight change}

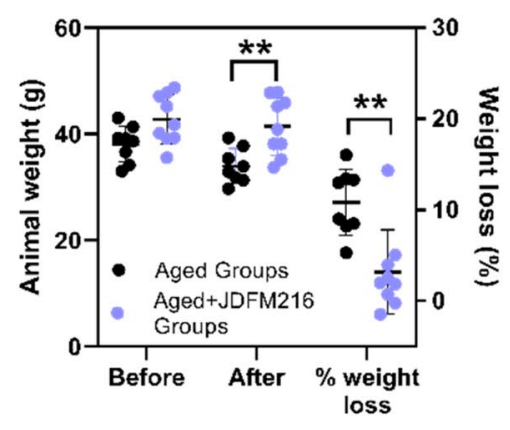

Figure 1. Behavioral assessments of JDFM216-treated aged mice. (A) Representation of the study design. JDFM216 or vehicle (sterile water) was administered for a total period of 48 weeks to C57BL/6 mice. (B) Y-maze test. (C) Voluntary wheel-running test. (D) Accelerated rotarod test. (E) Beam-walking test. (F) Forced swimming test (FST). (G) The average weight and \% weight loss of aged mice before and after the battery of behavioral tests (100 and 102 weeks). For all graphs, data are expressed as the means \pm SD. $n=13-15$ per group. Levels of significance determined by the Student's $t$ test are as follows: ${ }^{\star} P<0.05 ;{ }^{* *} P<0.01 ;{ }^{* *} P<0.001$, compared with the aged mouse group. 
suggesting that the probiotic administration of JDFM216 can help to improve the brain functions during the old age (Supplementary movie).

JDFM216 modulated the immune system of aged mouse. After the behavioral assessments, all mice were sacrificed to determine the immunoregulatory effects. Spleen samples were collected first; this observation was of interest as the mammalian spleen size has been correlated with differences in immune cell subsets and resistance to infection by encapsulated bacteria ${ }^{26}$. In our studies, compared with the Young Groups (8-weekold mice), the spleen indices in both of two aged mice groups (Aged Groups and Aged + JDFM216 Groups) showed a significant decrease (Fig. $2 \mathrm{~A}, \mathrm{P}<0.0001$ ), although there was no significant difference depending on the JDFM216 treatment. Next, the intestinal content of each mouse was collected from small intestine sections, and the level of secretory immunoglobulin A ( $\operatorname{Ig} \mathrm{A})$ production was determined. sIgA is the main immunoglobulin found in mucous secretions that plays a critical role in mucosal immunity protecting vulnerable areas such as the gut from invading pathogens ${ }^{27}$. In accordance with previous reports showing an increased level of sIgA by lactobacilli administration ${ }^{28,29}$, JDFM216 stimulated aged mice to produce a significantly high level of sIgA (over $10 \mathrm{ng} / \mathrm{mL} ; P=0.0015$ ) compared with the control group (Fig. 2B). Lymphocyte proliferation is commonly examined to assess the cell-mediated immunity when analyzing the efficacy of vaccines, diagnosis of infectious disease and immune deficiencies ${ }^{30}$. Approximately $5.0 \times 10^{6}$ splenocytes were separated from mice, and the proliferative capacity of splenic T-lymphocytes was measured. As a result, the JDFM216-treated group showed a significant increase in stimulation index $(S I)$ values over 4 days compared with the concanavalin A (ConA) control group (Fig. 2C; $\mathrm{P}<0.05$ ). These results indicate that JDFM216 can stimulate a T-lymphocyte proliferative response. To examine the phagocytic activity of peritoneal macrophages, quantitative spectrophotometric determination of neutral red accumulation in cells was measured in each group. It has been shown that stress stimulates the phagocytic capacity of macrophages preventing the entrance and survival of microorganisms in situation of immunodepression ${ }^{31}$. Figure 2D shows that the phagocytosis index $(P I)$ value in JDFM216-treated group was increased by nearly $30 \%$ compared with the control group $(P=0.0041)$. Dendritic cells play a pivotal immunoregulatory role with priming of the $\mathrm{T}$ helper cells, and probiotic bacteria are involved in modulation of the dendritic cell phenotype and function ${ }^{32-34}$. Splenocytes were separated from mice and used to investigate the effect of JDFM216 on CD11b and CD4 expression on the surface of dendritic cells by flow cytometry. Specifically, the percentage of $\mathrm{CD}_{11 \mathrm{~b}} \mathrm{CD}^{+}$cells in the JDFM216-treated group increased to $7.6 \%$, which was 2.7 -fold higher than the PBS control group (Fig. 2E; P<0.001), indicating that JDFM216 has the ability to stimulate the maturation of myeloid dendritic cells. The respiratory burst assay also showed that JDFM216 could activate mouse innate immunity, as seen in Fig. 2F. The leukocyte respiratory burst activity significantly increased in the JDFM216-treated group $(P=0.016)$, indicating that phagocytes enhanced the production of ROS by activating the NADPH oxidase enzyme that produces oxygen peroxide. In addition, we examined cytokines chemokine markers including IL-4, leukocyte recruitment (CXCL1), IL-10, IL-6 and TNF-alpha in mice serum (Fig. 2G,H and Fig. S1). These markers showed no significant differences, although a slight decrease was observed for IL-4 and CXCL1 with JDFM216 when compared with the controls. We also evaluated the cortisol level, but there was no significant difference between the control and JDFM216-supplemented groups (Fig. S1A). It is worth noting that the immunological measures were conducted after the behavioral tests which could be a potential acute stress to the mice, although the two groups of mice were treated in the same manner. The acute stress tends to induce leukocyte redistribution of cells and alter the innate immune responses, so the feasible confounding by the stress on the effects of immune responses could not be ruled out ${ }^{35}$.

Feeding JDFM216 influences the diversity of the gut microbiota in aged mice. To determine the impact of JDFM216 on the mouse gut microbiota, we analyzed the species richness and evenness. After normalizing the number of reads per sample, we obtained a total of 29,122 unique high-quality reads (14,000 reads per sample) that showed more than $99 \%$ Good's coverage for all samples (data not shown). First, comparison of the ecological indices showed that species richness (Chao index) and evenness (Shannon index) were higher in aged mice microbiota regardless of the JDFM216 treatment (Fig. S2), confirming the previous findings on increased diversity of gut microbiota with age in human and mice ${ }^{36-38}$. Results from Fig. 3A suggest that the aged mouse microbiota was significantly different from the young mouse microbiota $(\mathrm{P}<0.001)$. The differential abundance test indicated that OTUs belonging to the family Porphyromonadaceae and the genus Lactobacillus and Turicibacter were more abundant in young mice and that other OTUs belonging to the family Porphyromonadaceae and the genus Lactobacillus, Clostridium sensu stricto and Akkermansia were more abundant in old mice. The mean portion difference was highest for the Porphyromonadaceae OTU (Fig. 3B). Akkermansia muciniphila is a well-known mucin-degrading bacterium that is associated with anti-inflammatory effects in human. Similarly to our results, the bacterial composition tended to increase by aging possibly along with normal mucosa development ${ }^{39}$.

Among aged mice, we did not observe a significant shift in the microbiota by feeding JDFM 216 (Fig. 4A), but 4 and 7 OTUs were significantly increased and decreased by feeding JDFM216, respectively. Among them, OTU0023 Lactobacillus was increased by far the most, followed by OTU0090 Stenotrophomonas and OTU0099 Ruminococcaceae unclassified. The abundances of other OTUs belonging to the family Lachnospiraceae and Ruminococcaceae and the genus Ruminococcus were lower in the mice fed with JDFM216 (Fig. 4B). Stenotrophomonas is a genus of Gram-negative bacteria, comprising a broad range of species from beneficial probiotics $(S$. acidaminiphila) to opportunistic human pathogens (S. maltophilia $)^{40,41}$. In the view of gut microbiota, there is a report showing the enriched Stenotrophomonas in the Lactobacillus plantarum-treated group similar to our finding ${ }^{42,43}$. It is noted that the current $16 \mathrm{~S}$ metagenomics sequencing result indicates high abundance of Lactobacillus, probably present in the recipient intestines. In our previous study, we have shown that JDFM216 
(A)

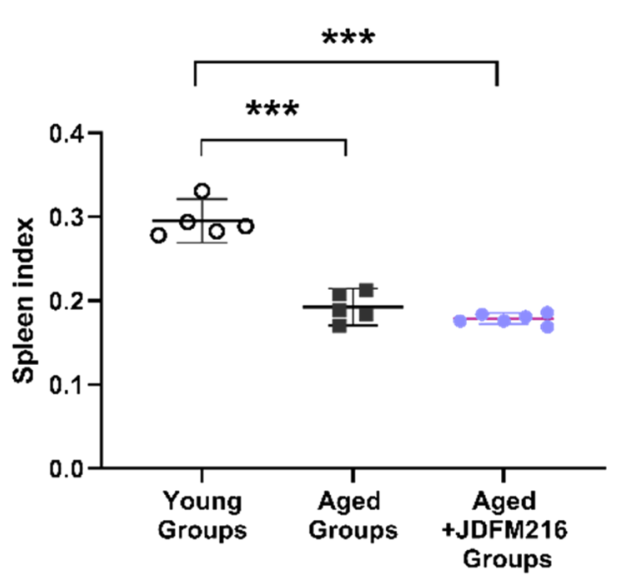

(C)

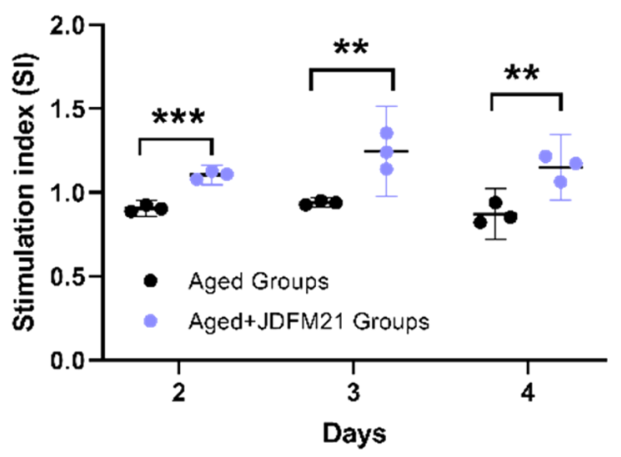

(E)

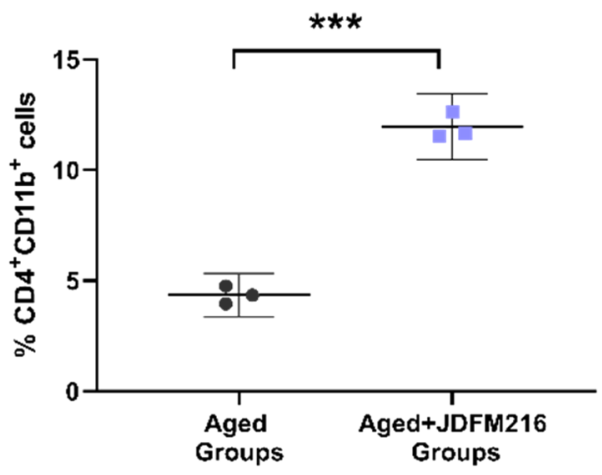

(G)

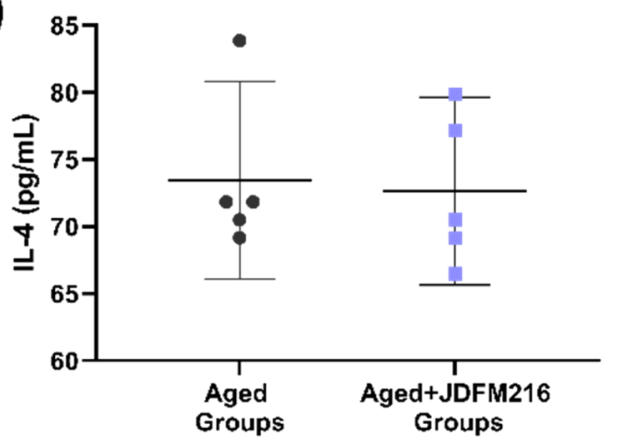

(B)

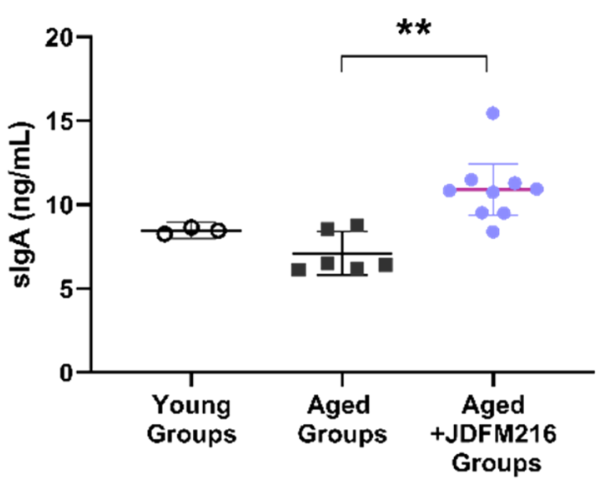

(D)

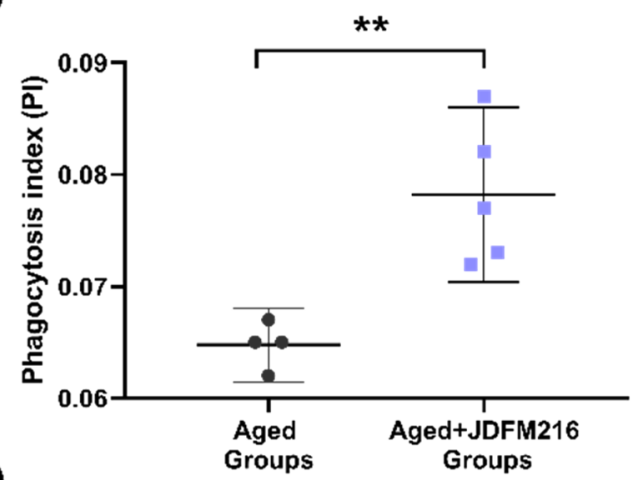

(F)

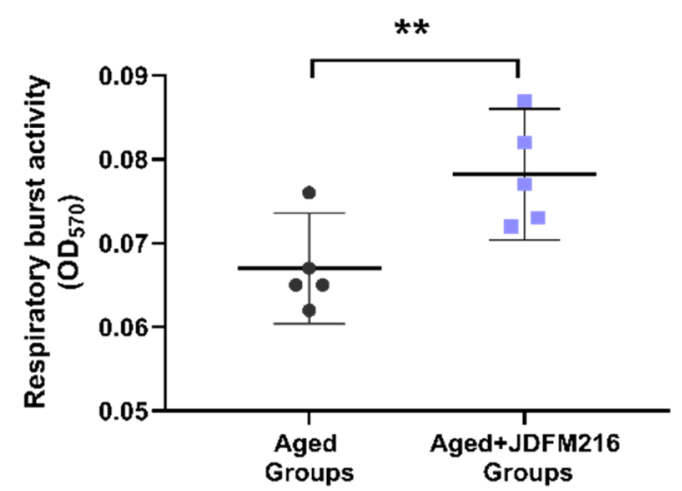

(H)

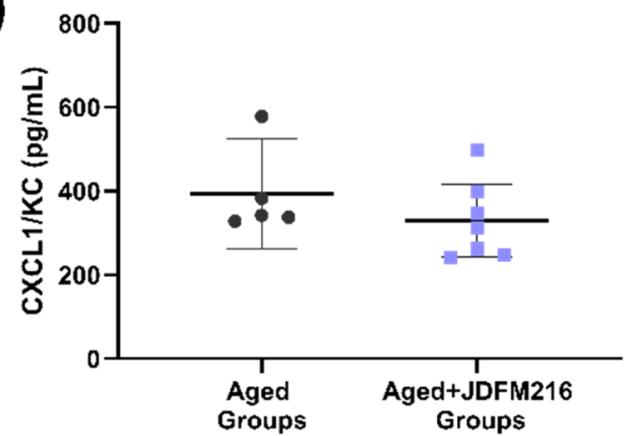

Figure 2. Profiling of the immune response in JDFM216-treated aged mice. (A) Spleen indices of young and aged mice (Young Groups, Aged Groups or Aged + JDFM216 Groups). (B) Secretary immunoglobulin A (sIgA) production in the intestine. (C) Proliferation of splenocytes. The proliferation was measured at 48, 72, and $96 \mathrm{~h}$ of cultivation. (D) Pinocytosis of peritoneal macrophages. (E) CD11b and CD4 expression on the surface of dendritic cells. (F) Leukocyte respiratory burst activity. Cytokine IL-4 and chemokine CXCL1 levels. (G,H). The young mouse group was used at 8 weeks of age. Values are means \pm SD. Asterisks represent statistically significant differences; one-way ANOVA was performed. ${ }^{\star} P<0.05 ;{ }^{* *} P<0.01 ;{ }^{* *} P<0.001$, compared with the groups. 
(A)

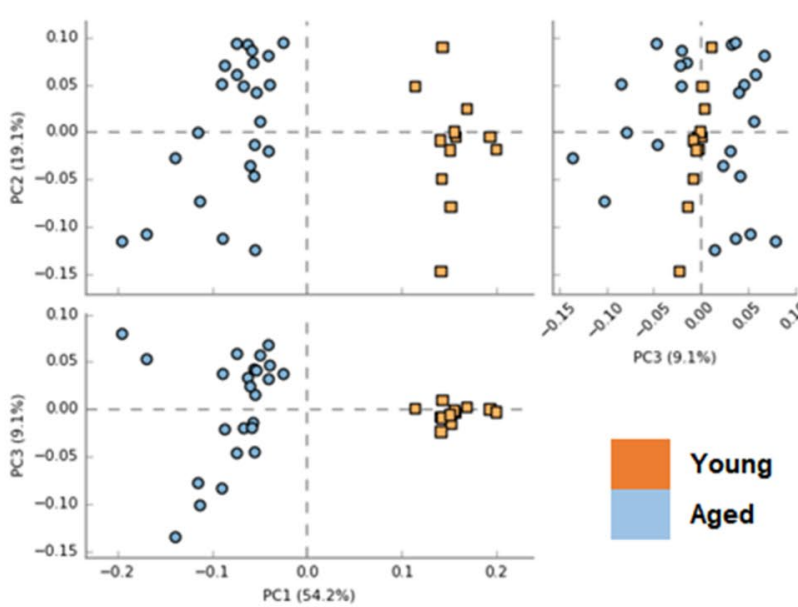

Mean portion difference $<0.1, p<0.01$

(B)

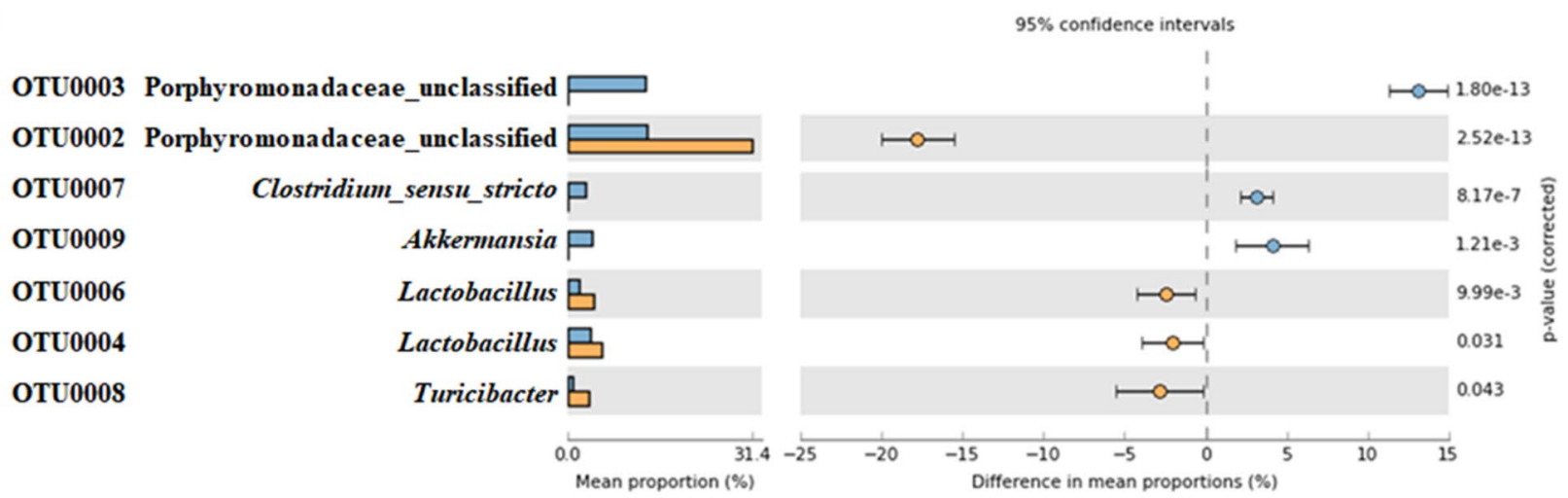

Figure 3. Comparison of gut microbiota between young mice (Young Groups at 8 weeks) and aged mice (Aged Groups at 100 weeks) based on principal component analysis (A) and operational taxonomic units (OTUs) with significantly different abundance $(\mathbf{B})$. Corrected $P$ values are shown on the right. The effect sizes and confidence intervals are provided to allow for critical assessment of the biological relevancy of the test results.

exhibits significantly high colonization ability to the intestinal tract of C. elegans ${ }^{44}$. Also, L. fermentum is known to possess an excellent adhesion ability in mice $^{45,46}$. In view of the findings, we speculate that JDFM216 would colonize the intestinal tract of the murine mouse model.

Next, we explored the metabolic activity of the gut microbiota in aged mice through PICRUSt analysis to predict the functional profiling of the microbial communities based on the 16S rRNA gene sequences. Interestingly, the mice in the aged groups showed a significant increase in the metabolic pathways of butanoate and propionate compared with the young groups (Fig. S3). Conversely, comparison of the metabolic activity between the aged and aged + JDFM216 groups did not show a dramatic difference (Fig. S4). The OTUs that showed a higher abundance among JDFM216-treated mice were further investigated to evaluate the association with metabolic activities estimated by PICRUSt 2 . These OTUs showed positive and negative moderate correlation $(-0.5<$ correlation $<0.5, \mathrm{p}<0.05)$ to 583 and 48 KEGG enzymes, respectively. Among them, the KEGG enzymes with a moderate correlation to the abundance of OTU0023 Lactobacillus are summarized in Table S1 and S2. Importantly, KEGG enzymes that were increased by feeding JDFM216 included vitamin B1, B2, and B6, butanoate, lipoic acid and taurine metabolism, all of which have been implicated in anti-aging effects. On the other hand, KEGG enzymes with a reduced abundance in the treated group included antibiotic metabolism. To support the functional profile prediction of OTU0023 Lactobacillus, we analyzed metabolites production of JDFM216 in the bacterial culture supernatant. As shown in Fig. S5, we could detect metabolic derivatives of butanoate including methyl-malonate, butanedioic acid, and butane-2,3-diol, of which abundances increased in the culture supernatant of JDFM216 compared to the blank medium. This suggests that JDFM216 supplementation would supply butanoate to the host.

\section{Discussion}

Aging accompanies severe chronic diseases that requires further investigation of mitigation approaches. There is now mounting evidence pointing to an important link among microbiota, behavior and the immune system ${ }^{47}$. Here we aimed to explore the impact of oral administration of JDFM216 on cognitive behaviors and immune responses in an aged murine model. We therefore designed intervention studies of JDFM216 for healthful 
(A)

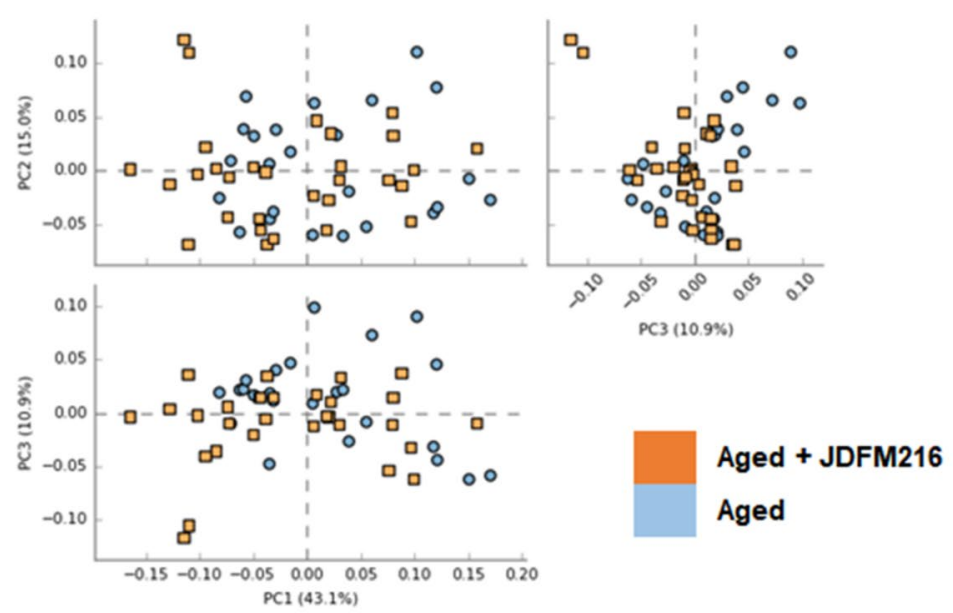

Mean portion difference $<0.1, \mathrm{p}<0.01$

(B)

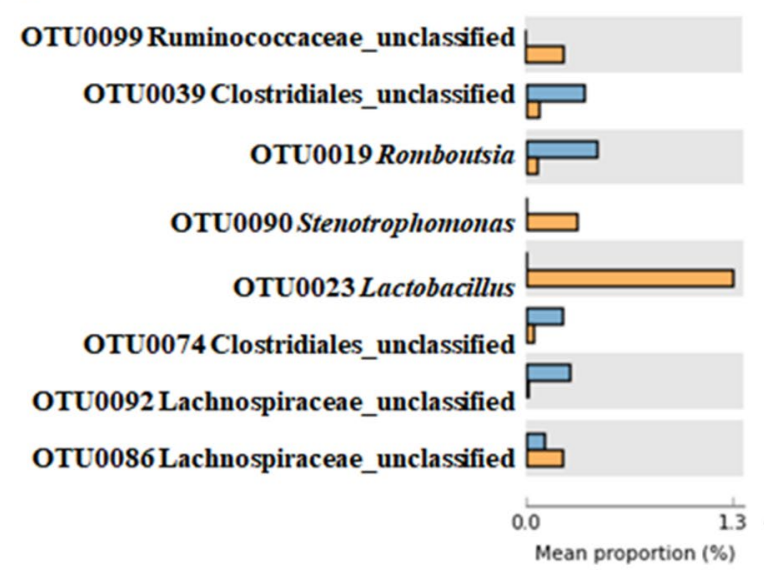

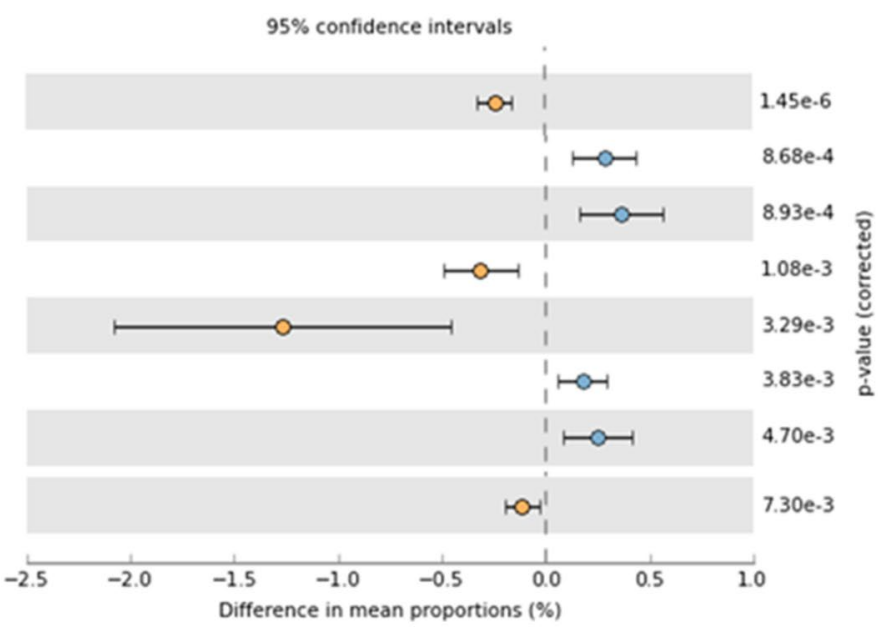

Figure 4. Comparison of gut microbiota between aged mice (Aged Groups) and aged mice fed Lactobacillus fermentum JDFM216 (Aged Groups + JDFM216) at 100 weeks based on principal component analysis (A) and operational taxonomic units (OTUs) with significantly different abundance (B). Corrected $P$ values are shown on the right. The effect sizes and confidence intervals are provided to allow for critical assessment of the biological relevance of the test results. Significantly increased (A) and decreased OTUs (B) and by feeding JDFM216.

longevity. L. fermentum JDFM216 has been studied for its health-promoting activities of longevity and immune response of the host, possessing phylogenetically featured genes related to the activities ${ }^{44,48}$.

As shown in the present study, JDFM216 induced a significant positive modulation of cognitive behaviors and physiological properties in aged mice. This result was supported by higher performances on Y-maze, accelerated rotarod, beam-walking and forced swimming tests with the administration of JDFM216. Other investigations have reported the effect of probiotics for improving memory in middle-aged rats as a therapeutic agent using the Morris water maze and Pavlovian autoshaping tests ${ }^{49}$. The anti-depressant effect of probiotics has been demonstrated in animal models by the FST and tail suspension test (TST) ${ }^{50,51}$. In addition to these reports, the cognitive functions being altered by probiotics are mostly psychiatric disorders and memory abilities, which were confirmed in the current study ${ }^{52}$. Our results of the JDFM216 treatment also suggested improvements of motor performance and balance ability. The improvement of spatial working memory by the Y-maze test might be implicated by the hyperlocomotion which could significantly interfere with the measurement of spontaneous alternation ${ }^{53}$. Also, the forced swimming test has disadvantages on lacking sufficient mechanistic specificity, one-dimensional outcome and influencing behaviors by repeated trials. To specify and validate the behavioral phenotypes induced by the probiotics, further behavioral tests are required.

In this study, we observed stimulatory effects of JDFM216 immune system, such as enhanced phagocytic activity of macrophages and secreted intestinal sIgA. Beneficial effects of probiotics on the host intestinal immune functions have been identified, including modulation of innate immunity and priming of the humoral adaptive immune system ${ }^{54,55}$. Probiotics also play a role in maintaining the balance between innate and adaptive immune responses by directly interacting with intestinal epithelial cells or interacting with dendritic cells and follicleassociated epithelial cells via $M$ cells, further initiating responses mediated by macrophages and lymphocytes ${ }^{55}$. 
These processes can be mediated by probiotics through inducing the production of different chemokines and modulating intestinal microbiota ${ }^{56,57}$. Our results support these current findings on the stimulatory effects of probiotics on the host immune modulation, however, we could not verify the specific cytokines expression affected by JDFM216 in the current study. We also measured the cortisol level in mice serum, but there was no significant difference between the two groups. Although the corticosterone is a main hormone involved in stress responses with an association of fear memory recognition, a recent study reported that circulating glucocorticoids are mostly bound to corticosteroid-binding globulin, and only approximately $5 \%$ of the hormones can cross the blood-brain barrier as free plasma glucocorticoids ${ }^{58}$. They also showed that intrahippocampal corticosterone generation was more important factor in stress-associated brain function than the blood corticosterone level. Therefore, it is still possible that the aged control mice would be more vulnerable to stress, although the blood corticosterone level was not significantly different to that of JDFM216-supplemented mice. In order to better understand the molecular mechanisms of JDFM216 that link the gut microbiota, immune, and central nervous systems in a network communication, further investigation on a broader range of immune responses will be conducted.

This study demonstrated the difference of gut microbiota composition between young and aged mice, and that treatment of JDFM216 was able to modulate the abundance of some OTUs including Lactobacillus itself. Previous studies have reported that administration of Lactobacillus strains modulates the gut microbiota and alleviates aging-induced metabolic diseases ${ }^{59,60}$. In this study, we observed that JDFM216 treatment significantly and mostly increased one OTU belonging to the genus Lactobacillus, the abundance of which was correlated to vitamin metabolism and other beneficial substances such as butanoate, lipoic acid, and taurine. Butanoate is probably the most reported beneficial metabolite produced by intestinal bacteria. It has been reported that consumption of conventional yogurt increases plasma vitamin B1, B2 and B6 ${ }^{61}$, the metabolism of which was correlated to significantly increased Lactobacillus OTU by feeding with JDFM216. Lipoic acid is also known for its anti-aging properties through reducing oxidative stress ${ }^{62}$ and preventing memory loss ${ }^{63}$. Taurine is also known to increase hippocampal neurogenesis in aging mice ${ }^{64}$. These results suggest that such microbial production of functional metabolites may ultimately target diseases linked to aging and inflammation in host-microbial interactions.

The present study identifies associations of the gut microbiota, host immune system, and cognitive functions with respect to oxidative stress by increased phagocytic capacity, leukocyte respiratory burst activity, microbial metabolic products, and possibly altered cognitive behaviors. Oxidative stress has been implicated in a wide variety of degenerative processes including aging. It induces protein and DNA damage in conjunction with genetic instability and insufficient DNA damage repair ${ }^{65}$. Butanoate, lipoic acid, vitamin B6 and taurine, which are positively correlated with the OTU of Lactobacillus, are well known to have antioxidative effects by preventing oxygen radical generation ${ }^{66}$, antagonizing electrophilic oxidative stress ${ }^{67}$, and activating the transcription factor Nrf-2 (NF-E2 p45-related factor 2), which is a master regulator of cellular detoxification responses and redox status $^{17,68}$. Recently, a number of studies have been published showing that probiotics have potent antioxidant capacity through the Nrf2 signaling pathway ${ }^{69,70}$. In addition, our previous studies revealed that the healthpromoting effects of JDFM216 were mediated by a nuclear hormone receptor family and PMK-1 signaling cascade which regulates the oxidative stress response in C. elegans ${ }^{44,48}$. Further detailed mechanisms underlying gut bacteria-derived metabolite and aging-related antioxidant effects remain to be determined.

Taken together, administration of the probiotic L. fermentum strain JDFM216 in aged mice confirmed its features of the health promotion on the cognitive host responses and the immune system through the alteration of the intestinal taxonomic composition. The findings facilitate our understanding on the efficacy of probiotics and probiotic-derived factors on the regulation of the host psychological and immune health, thus providing a fascinating potential for probiotic application in human disease prevention and treatment.

\section{Methods}

Bacterial strains and growth conditions. Lactobacillus fermentum JDFM216, previously isolated from infant feces (referred to as JDFM216; Jang et al., 2017) was grown in Man, Rogosa and Sharpe (MRS) medium overnight at $37^{\circ} \mathrm{C}$. To prepare live bacterial feeding, strains were washed twice with sterile water and suspended in sterile water at a concentration of $c a 1.0 \times 10^{9} \mathrm{CFU} / \mathrm{mL}$.

Animals and experimental design. All mice were 6-week-old male C57BL/6 mice purchased from Orient Bio Inc. (Seongnam, Korea). Groups of 13-15 mice (randomly assigned to control or experimental groups) were housed in isolator cages (JD-C-07, Jeungdo Bio \& Plant Co., Seoul, Korea) with sterilized bedding under the following auto-controlled conditions: lighting ( $12 \mathrm{~h}$ light and $12 \mathrm{~h}$ dark), temperature $\left(24{ }^{\circ} \mathrm{C}\right)$, and relative humidity (55\%). The acclimatization period was 2 weeks. Because C57BL/6 male mice have a long life span of approximately 26 to 28 months and are widely used for gerontological studies, we employed 12-month-old (52 weeks) mice of this strain as a model of middle-aged mice, and fecal and blood samples were first collected at 8 weeks for comparison with young mice (Young Groups). Figure 1A represents a schematic of the experimental plan. Initially, for the aging model of middle-aged mice, all mice maintained in sterile isolators and fed irradiated normal chow and water ad libitum for a total of 52 weeks. Then, sterile water was administered with or without living cells of JDFM216 (approximately $6.0 \times 10^{8} \mathrm{CFU} / \mathrm{mice} /$ day) for 48 weeks to ensure that senescence was sufficiently developed (Aged Groups vs. Aged + JDFM216 Groups). At the indicated weeks (63, 77, and 100 weeks) during the administration period, fecal or blood samples were collected for analyzing fecal microbiota and biological features. To reduce stress during the long-term period of the experiment, all water administration was given freely, and the behavior tests were conducted sequentially from the least to the most stressful tasks. These tasks were designed for the measurement of spontaneous activity, short-term memory, motor function, locomotor activity, and depression level. All experiments were handled in accordance with institutional ethical guide- 
lines, and this study was approved by the Institutional Animal Care and Use Committees (IACUC) of Jeonbuk National University (2015-05).

Behavioral assessments. Y-maze test. For the measurement of learning and memory abilities, the Ymaze test was performed according to a method described previously ${ }^{71}$. The Y-maze apparatus consisted of a three-arm horizontal maze (35-cm-long and 5-cm-wide with $15-\mathrm{cm}$-high walls) in which the arms are symmetrically disposed at $120^{\circ}$ angles from a central platform. Mice were initially placed at the end of one arm and allowed to explore freely. The number of arm entries serves as an indicator of locomotor activity, and its sequence was recorded manually for each mouse over an $8-\mathrm{m}$ period. An actual alternation was defined as entries into all three arms for consecutive choices. The percentage of alternations was defined by the following equation: $\%$ Alternation $=[($ Number of actual alternations $) /($ Total arm entries -2$)] \times 100$.

Voluntary wheel-running test. All mice for the test were individually housed in cages containing running wheels. The running wheels consisted of a rotating metal chamber with a wire mesh floor $(32 \mathrm{~cm}$ in diameter, $10 \mathrm{~cm}$ in width) attached to a stationary metal wall. Mice were trained for 20 min per day for three consecutive days inside a wheel. During the training session, the animal was free to run but could not leave the wheel. Distance (yards) of rotations was monitored for $300 \mathrm{~s}$, and scores of individual profiles were averaged for each group.

Accelerated rotarod test. Evaluation of motor ability was performed using the accelerating treadmill rotarod instrument (BS Technolab) with automatic timers and falling sensors as described earlier ${ }^{72}$. The apparatus consisted of a black striated rod $(3 \mathrm{~cm}$ in diameter) and $25 \mathrm{~cm}$ above the floor. Before the training sessions, the mice were habituated to stay on the stationary drum for $5 \mathrm{~min}$. Habituation was repeated every day for $1 \mathrm{~min}$ immediately before the session. Mice were trained for one daily session consisting of three trials over three consecutive days. On the first day, the rotation speed was set at a fixed value of $10 \mathrm{rpm}$ for $300 \mathrm{~s}$. When an animal fell off during this period, it was placed back on the rod until the end of the trial. The speed for the second and third days was set to 20 and $30 \mathrm{rpm}$. After the last exposure, the rod was then set to accelerate from 4 to $40 \mathrm{rpm}$ over a period of $300 \mathrm{~s}$, and the time spent on the drum was recorded automatically for each mouse. Trials within the same day were performed with a 15-min rest period between trials. After testing was finished, the rod was cleaned and dried to ensure a non-slippery surface for the following animal. The data reported for each mouse are the average latency to fall from the rod for the three trials.

Balance beam test. This test measured the ability of the mice to traverse a progressive series of narrow wooden beam as described earlier ${ }^{73}$. The beam was made of wood and consisted of a square section that was $1 \mathrm{~m}$ in length and suspended horizontally $80 \mathrm{~cm}$ from the floor. Mice were trained over 2 days with three trials per day on the square beam ( $40 \mathrm{~mm}$ wide) until they could cross the beam quickly and without stopping. After training, each mouse was forced to walk along a narrow square beam $(30 \mathrm{~mm}$ wide) to reach the end of the beam. The measurements taken were the number of foot slips and recorded with a maximum time of $300 \mathrm{~s}$. The recorded data were scored as follows: 0 , does not attempt to balance; 1 , on the beam without moving; 2 , turns on the left or right; 3 , slipped over $50 \%$ while crossing; 4 , slipped from two times to below $50 \%$ while crossing; 5 , slipped only one time while crossing; and 6 , crossed the beam without slips. For tests, scoring was carried out using video-tracking.

Forced swimming test (FST). Depression behavior was assessed using the forced swim test as previously described $^{74}$, which is a standard rodent test for screening antidepressant activity. The FST is one of the most used tools in preclinical research on depression treatment because it is a low-cost, fast, and reliable animal model with strong predictive validity. The experimental protocol requires mice to be placed in a water-filled large cylinder. The FST apparatus consisted of a transparent cylinder $(10 \mathrm{~cm}$ diameter, $28 \mathrm{~cm}$ height $)$ filled with water $(18 \mathrm{~cm}$, $24 \pm 2{ }^{\circ} \mathrm{C}$ ). During their first experience, mice were exposed to FST for $10 \mathrm{~min}$ (training). Twenty-four hours later, all the mice were exposed to the apparatus (test). The tests were assessed in a 6-min period, and the duration of immobility within the last $4 \mathrm{~min}$ of the test was recorded. The depression level is scored for immobile behavior, defined as stops struggling and floats motionless on the water, making only movements to maintain its head above water.

Immunomodulatory assays. Detection of spleen indices. The body weight of mice was recorded, and then the mice were sacrificed by cervical dislocation. Spleens were obtained from the mice and weighed immediately. The spleen indices were measured as the ratio of the spleen weight to the mouse body weight.

Measurement of intestinal sIgA. After sacrificing the mice, intestinal samples from $5 \mathrm{~cm}$ of the cecum and distal sections of the intestine were collected and washed with PBS. The samples were homogenized in $0.5 \mathrm{~mL}$ PBS containing $1 \%$ protease inhibitor cocktail. The homogenized solution was centrifuged at 13,000 rpm for $15 \mathrm{~min}$ at $4{ }^{\circ} \mathrm{C}$ to remove particulate material. The supernatant was collected and assayed using commercially available ELISA kits for mouse secretory immunoglobulin A (sIgA) (Cat No. MBS269144, MyBioSource, San Diego, CA, USA) for sIgA determination.

Splenocyte proliferation assay. The splenocytes of each mouse groups were aseptically isolated using $70 \mu \mathrm{m}$ cell strainer, washed and resuspended in ammonium chloride-Tris (ACT) buffer and deposited in 96-well plates at a concentration of $5.0 \times 10^{6}$ cells/well with concanavalin A (ConA, $\left.2 \mathrm{mg} / \mathrm{mL}\right)$. Splenocytes incubated with medium alone used as negative control. After cultivating Splenocytes and ConA for $96 \mathrm{~h}$ at $37^{\circ} \mathrm{C}$ under atmosphere of $5 \%$ 
$\mathrm{CO}_{2}, 20 \mu \mathrm{L}$ of MTS solution (Promega, Madison, WI, USA) was treated. And then, the plate was incubated for additional $2 \mathrm{~h}$ and read at $490 \mathrm{~nm}\left(\mathrm{OD}_{490}\right)$ using a Synergy HTX Hybrid Multi Mode Microplate Reader (BioTek Instruments, Inc., Winooski, VT, USA). The proliferation of splenocyte were calculated by the stimulation index (SI): $\mathrm{SI}=\mathrm{OD}_{490}$ of stimulated cells $/ \mathrm{OD}_{490}$ of the negative control.

Determination of pinocytosis of peritoneal macrophage. In the present study, mice were sacrificed and peritoneal cells were cautiously collected by peritoneal lavage with PBS. Then, $3 \mathrm{~mL}$ of lavage fluid was harvested and centrifuged at $2000 \mathrm{rpm}$ for $10 \mathrm{~min}$. The collected cells were resuspended with the concentrations of $1 \times 10^{6}$ cells/ $\mathrm{mL}$ in RPMI1640 medium and located on $200 \mu \mathrm{L}$ per well in 96-well plates. After culturing for $24 \mathrm{~h}$ at $37^{\circ} \mathrm{C}$, the medium was discarded, and then $100 \mu \mathrm{L}$ of $0.072 \%$ neutral red solution was exposed to separate wells. Following additional cultivation for $30 \mathrm{~min}$, each well was carefully washed three times with DPBS buffer. Next, $100 \mu \mathrm{L}$ of lysis solution consisted of acetic acid and alcohol $(1: 1 ; \mathrm{v} / \mathrm{v})$ was treated to each well, read at $570 \mathrm{~nm}\left(\mathrm{OD}_{570}\right)$ using microplate reader and finally evaluated as the phagocytosis index (PI). The PI was calculated according to the following formula: $\mathrm{PI}=$ (total number of engulfed cells/total number of counted macrophages $) \times($ number of macrophages containing engulfed cells/total number of counted macrophages $) \times 100$.

Serum analysis. Blood samples were collected after the mice were sacrificed. The fresh blood was kept standing for $60 \mathrm{~min}$ at $37^{\circ} \mathrm{C}$. Serum was obtained by centrifugation at $3000 \mathrm{rpm}$ for $15 \mathrm{~min}$ and then stored at $-80^{\circ} \mathrm{C}$ until use. Concentrations of cytokines including IL-4, IL-6, IL-10, CXCL1/KC, and TNF- $\alpha$ in serum were determined using a magnetic Luminex screening assay according to the manufacturer's instructions (R\&D Systems, Minneapolis, MN) on a Bio-Plex 200 analyzer (Bio-Rad, Hercules, CA). In addition, serum corticosterone level was measured using the corticosterone (Abnova, Taipei, Taiwan) and serotonin (Abcam, Cambridge, MA, USA) enzyme-linked immunosorbent assay kits, respectively, according to the manufacturer's instructions.

$C D 11 b^{+} C D 4^{+}$lymphocyte subset analysis. The percentage of lymphocyte subpopulations in the spleen was measured using flow cytometric analysis. Approximately $9.0 \times 10^{7}$ cells $/ \mathrm{mL}$ of splenocytes were stained for $\mathrm{CD}^{+}$ cell isolation using the MACS Treg isolation kit (Miltenyi Biotec, CA), and then they were separated using CD $11 \mathrm{~b}^{+}$microbeads and a MACS LS column (Miltenyi Biotec, CA) according to the manufacturer's directions. The mixed samples were washed twice with fluorescence-activated cell sorting (FACS) buffer (PBS, 1\% FCS, and $0.09 \%$ sodium azide) and analyzed by flow cytometry (BD FACSCalibur). CD $4^{+}$CD $11 b^{+}$cells were expressed as proportion of cells positive for the markers per the number of total splenocytes.

Respiratory burst assay. The released amount of total $\mathrm{O}_{2}^{-}$from monocytes was determined as superoxide dismutase (SOD)-inhibitable reduction of JDFM216 at $570 \mathrm{~nm}$ in microtiter plates. The assay was carried out following a previous established method with modifications in the detection wavelength ${ }^{75}$. The method consists of a colorimetric determination of the ROS produced by the leukocyte respiratory burst, which promotes the reduction of nitroblue tetrazolium into a dark blue precipitate inside the phagocyte, called formazan granules. The cells were stimulated with PMA or PBS. Values in the absence of stimulus were subtracted from those obtained after stimulation.

16S rRNA sequencing metagenomic analysis. Fecal microbiota sequencing. Total DNA was extracted from $100 \mathrm{mg}$ of feces collected from each mouse. The V4 region of the $16 \mathrm{~S}$ ribosomal RNA gene was amplified by the polymerase chain reaction (PCR) using specific primers as previously described ${ }^{76}$. Following purification of the PCR products, individual indices were added to the amplicons by PCR. The amplicons were then sequenced using the Illumina MiSeq platform according to the manufacturer's instructions. The replicates of the DNA samples were pooled and subjected to amplicon sequencing using MiSeq according to the manufacturer's instructions. All sequencing was performed at Macrogen Inc. (Seoul, Republic of Korea).

Microbial community analysis. The bacterial composition and amplicon sequence analysis were conducted according to MiSeq SOP (https://www.mothur.org/wiki/MiSeq_SOP). Briefly, MOTHUR ${ }^{77}$ was used to assemble, align, and cluster the reads. Alignment was performed based on the database from SILVA (version 132) ${ }^{78}$, and taxonomic classification was performed based on the Ribosomal database project database ${ }^{79}$ and clustered based on OptiClust algorithms ${ }^{80}$ with a sequence distance 0.03 . Chimeric sequences were removed using $\mathrm{VSEARCH}^{81}$. The distribution of operational taxonomic units (OTUs) was analyzed using STAMP ${ }^{81}$ based on the distance calculated using the Bray-Curtis coefficient. Gut microbiota derived metabolic activities were estimated using PICRUSt2 (https://github.com/picrust/picrust2). Functional pathways were annotated from Kyoto Encyclopedia of Genes and Genomes (KEGG, https://www.genome.jp) databases ${ }^{82,83}$.

Metabolites analysis. Culture supernatant of L. fermentum JDFM216 grown overnight in MRS were collected by centrifugation at $12,000 \mathrm{rpm}$ for $10 \mathrm{~min}$ at $4{ }^{\circ} \mathrm{C}$, followed by filtration through a $0.2-\mu \mathrm{m}$ PVDF membrane. Extracellular metabolites were extracted by adding the culture supernatant to ice-cold methanol and vortexed for $1 \mathrm{~min}$. After centrifugation at $10,000 \mathrm{rpm}$ for $10 \mathrm{~min}$ at $4{ }^{\circ} \mathrm{C}$, the upper layer of the supernatant was collected, concentrated to dryness in a vacuum concentrator, and stored at $-80^{\circ} \mathrm{C}$ prior to derivatization and analysis by GC-MS.

The extract was derivatized with $60 \mu \mathrm{L}$ of a solution of $20 \mathrm{mg} / \mathrm{mL}$ methoxyamine hydrochloride in pyridine (Sigma, St. Louis, MO, USA) at $30^{\circ} \mathrm{C}$ for $90 \mathrm{~min}$, and $100 \mu \mathrm{L}$ of N,O-Bis(trimethylsilyl)trifluoroacetamide (BSTFA; Sigma) was subsequently added at $60{ }^{\circ} \mathrm{C}$ for $30 \mathrm{~min}$. A mixture of fatty acid methyl esters 
and fluoranthene was added to the extract as internal standards. The GC-MS analysis was conducted using a Thermo Trace 1310 GC (Waltham, MA, USA) coupled to a Thermo ISQ LT single quadrupole mass spectrometer (Waltham, MA, USA). A DB-5MS column with 60-m length, $0.2-\mathrm{mm}$ i.d. and $0.25-\mu \mathrm{m}$ film thickness (Agilent, Santa Clara, CA, USA) was used for separation. For analysis, the sample was injected at $300{ }^{\circ} \mathrm{C}$ and split ratio 1:5 with $7.5 \mathrm{~mL} / \mathrm{min}$ helium split flow. The metabolites were separated with $1.5 \mathrm{~mL}$ constant flow helium with an oven ramp of $50{ }^{\circ} \mathrm{C}(2 \mathrm{~min}$ hold $)$ to $180^{\circ} \mathrm{C}(8 \mathrm{~min}$ hold $)$ at $5^{\circ} \mathrm{C} / \mathrm{min}$, to $210^{\circ} \mathrm{C}$ at $2.5^{\circ} \mathrm{C} / \mathrm{min}$, and to $325^{\circ} \mathrm{C}$ ( $10 \mathrm{~min}$ hold) at $5^{\circ} \mathrm{C} / \mathrm{min}$. The mass spectra were acquired in a scan range of $35-650 \mathrm{~m} / \mathrm{z}$ at an acquisition rate of 5 spectra per sec. The ionization mode was subjected to electron impact, and the temperature for the ion source was set to $270^{\circ} \mathrm{C}$. The spectra were processed by Thermo Xcalibur software (version 4.1, Waltham, MA, USA), using automated peak detection, and the metabolites were identified by matching the mass spectra and retention indices of the NIST Mass spectral search program (version 2.0, Gaithersburg, MD, USA).

Statistics. Data were analyzed using GraphPad Prism software (version 8, San Diego, CA, USA). All results are presented as the mean \pm standard deviation (SD). Statistical analysis was performed using the Student's $t$ test for comparisons between two groups and one-way analysis of variance for comparisons among multiple groups. $P<0.05$ was considered significant. Spearman correlation analysis was applied to identify correlated OTUs to metabolic activities. Significant differences $(P<0.05)$ between samples were estimated at the OTU level.

Received: 28 May 2020; Accepted: 10 November 2020

Published online: 10 December 2020

\section{References}

1. Sehl, M., Sawhney, R. \& Naeim, A. Physiologic aspects of aging: impact on cancer management and decision making, part II. Cancer J. 11, 461-473 (2005).

2. Bäckhed, F. et al. The gut microbiota as an environmental factor that regulates fat storage. Proc. Natl. Acad. Sci. U.S.A. 101, 15718-15723 (2004).

3. Aleman, F. D. D. \& Valenzano, D. R. Microbiome evolution during host aging. PLoS Pathog. 15, e1007727 (2019).

4. Cryan, J. F. \& Dinan, T. G. Mind-altering microorganisms: the impact of the gut microbiota on brain and behaviour. Nat. Rev. Neurosci. 13, 701-712 (2012).

5. Sommer, F. \& Bäckhed, F. The gut microbiota-masters of host development and physiology. Nat. Rev. Microbiol. 11, 227-238 (2013).

6. Diaz Heijtz, R. et al. Normal gut microbiota modulates brain development and behavior. Proc. Natl. Acad. Sci. U.S.A. 108, 3047$3052(2011)$.

7. Cryan, J. F., O’Riordan, K. J., Sandhu, K., Peterson, V. \& Dinan, T. G. The gut microbiome in neurological disorders. Lancet Neurol. 19, 179-194 (2020).

8. Kouchaki, E. et al. Clinical and metabolic response to probiotic supplementation in patients with multiple sclerosis: a randomized, double-blind, placebo-controlled trial. Clin. Nutr. 36, 1245-1249 (2017).

9. Russo, A. J. Decreased plasma myeloperoxidase associated with probiotic therapy in autistic children. Clin. Med. Insights Pediatr. 9, 13-17 (2015).

10. Unger, M. M. et al. Short chain fatty acids and gut microbiota differ between patients with Parkinson's disease and age-matched controls. Parkinsonism Relat. Disord. 32, 66-72 (2016).

11. Akbari, E. et al. Effect of probiotic supplementation on cognitive function and metabolic status in Alzheimer's disease: a randomized, double-blind and controlled trial. Front. Aging Neurosci. 8, 256 (2016).

12. Naveed, M. et al. Gut-brain axis: a matter of concern in neuropsychiatric disorder. Prog. Neuropsychopharmacol. Biol. Psychiatry 104, 110051 (2021).

13. Nishida, K., Sawada, D., Kuwano, Y., Tanaka, H. \& Rokutan, K. Health benefits of Lactobacillus gasseri CP2305 tablets in young adults exposed to chronic stress: a randomized, double-blind, placebo-controlled study. Nutrients 11, 1859 (2019).

14. Rao, A. V. et al. A randomized, double-blind, placebo-controlled pilot study of a probiotic in emotional symptoms of chronic fatigue syndrome. Gut Pathog. 1, 6 (2009).

15. Brüssow, H. Microbiota and healthy ageing: observational and nutritional intervention studies. Microb. Biotechnol. 6, 326-334 (2013).

16. Sharma, R. et al. Diet supplemented with phytochemical epigallocatechin gallate and probiotic Lactobacillus fermentum confers second generation synbiotic effects by modulating cellular immune responses and antioxidant capacity in aging mice. Eur. J. Nutr. 58, 2943-2957 (2019).

17. Chen, C. et al. Roles of taurine in cognitive function of physiology, pathologies and toxication. Life Sci. 231, 116584 (2019).

18. Messaoudi, M. et al. Assessment of psychotropic-like properties of a probiotic formulation (Lactobacillus helveticus R0052 and Bifidobacterium longum R0175) in rats and human subjects. Br. J. Nutr. 105, 755-764 (2011).

19. Sanborn, V., Azcarate-Peril, M. A., Updegraff, J., Manderino, L. M. \& Gunstad, J. A randomized clinical trial examining the impact of LGG probiotic supplementation on psychological status in middle-aged and older adults. Contemp. Clin. Trials Commun. 12, 192-197 (2018).

20. Corpuz, H. M. et al. Long-term diet supplementation with Lactobacillus paracasei K71 prevents age-related cognitive decline in senescence-accelerated mouse prone 8. Nutrients 10, 762 (2018).

21. Distrutti, E. et al. Modulation of intestinal microbiota by the probiotic VSL\#3 resets brain gene expression and ameliorates the age-related deficit in LTP. PLoS ONE 9, e106503 (2014).

22. Dutta, S. \& Sengupta, P. Men and mice: relating their ages. Life Sci. 152, 244-248 (2016).

23. Albani, S. H., McHail, D. G. \& Dumas, T. C. Developmental studies of the hippocampus and hippocampal-dependent behaviors: insights from interdisciplinary studies and tips for new investigators. Neurosci. Biobehav. Rev. 43, 183-190 (2014).

24. Woo, J.-Y. et al. Lactobacilluspentosus var. plantarum C29 ameliorates memory impairment and inflammaging in a D-galactoseinduced accelerated aging mouse model. Anaerobe 27, 22-26 (2014).

25. Wong, A. A. \& Brown, R. E. Age-related changes in visual acuity, learning and memory in C57BL/6J and DBA/2J mice. Neurobiol. Aging 28, 1577-1593 (2007).

26. Hadidi, S., Glenney, G. W., Welch, T. J., Silverstein, J. T. \& Wiens, G. D. Spleen size predicts resistance of rainbow trout to Flavobacterium psychrophilum challenge. J. Immunol. 180, 4156-4165 (2008).

27. Corthésy, B. Multi-faceted functions of secretory IgA at mucosal surfaces. Front. Immunol. 4, 185 (2013) 
28. Xu, H. et al. Oral administration of compound probiotics improved canine feed intake, weight gain, immunity and intestinal microbiota. Front. Immunol. 10, 666 (2019).

29. Hu, J. et al. Lactobacillus frumenti facilitates intestinal epithelial barrier function maintenance in early-weaned piglets. Front. Microbiol. 9,897 (2018).

30. Nikbakht, M., Pakbin, B. \& Nikbakht Brujeni, G. Evaluation of a new lymphocyte proliferation assay based on cyclic voltammetry; an alternative method. Sci. Rep. 9, 4503 (2019).

31. Ferreira, C. K. O. et al. Phagocytic responses of peritoneal macrophages and neutrophils are different in rats following prolonged exercise. Clinics (Sao Paulo) 65, 1167-1173 (2010).

32. Foligne, B. et al. A key role of dendritic cells in probiotic functionality. PLoS ONE 2, e313 (2007).

33. Christensen, H. R., Frøkiaer, H. \& Pestka, J. J. Lactobacilli differentially modulate expression of cytokines and maturation surface markers in murine dendritic cells. J. Immunol. 168, 171-178 (2002).

34. Hart, A. L. et al. Modulation of human dendritic cell phenotype and function by probiotic bacteria. Gut 53, 1602-1609 (2004).

35. Bowers, S. L., Bilbo, S. D., Dhabhar, F. S. \& Nelson, R. J. Stressor-specific alterations in corticosterone and immune responses in mice. Brain Behav. Immun. 22, 105-113 (2008).

36. Hoffman, J. D. et al. Age drives distortion of brain metabolic, vascular and cognitive functions, and the gut microbiome. Front. Aging Neurosci. 9, 298 (2017)

37. An, J. Y. et al. Rapamycin rejuvenates oral health in aging mice. Elife $\mathbf{9}$, e54318 (2020).

38. Biagi, E. et al. Gut microbiota and extreme longevity. Curr. Biol. 26, 1480-1485 (2016).

39. Collado, M. C., Derrien, M., Isolauri, E., de Vos, W. M. \& Salminen, S. Intestinal integrity and Akkermansia muciniphila, a mucindegrading member of the intestinal microbiota present in infants, adults, and the elderly. Appl. Environ. Microbiol. 73, 7767-7770 (2007).

40. Zhang, Q., Saleem, M. \& Wang, C. Probiotic strain Stenotrophomonas acidaminiphila BJ1 degrades and reduces chlorothalonil toxicity to soil enzymes, microbial communities and plant roots. AMB Express 7, 227 (2017).

41. Svensson-Stadler, L. A., Mihaylova, S. A. \& Moore, E. R. B. Stenotrophomonas interspecies differentiation and identification by gyrB sequence analysis. FEMS Microbiol. Lett. 327, 15-24 (2012).

42. Liu, G. et al. Lactic acid bacteria feeding reversed the malformed eye structures and ameliorated gut microbiota profiles of Drosophila melanogaster Alzheimer's disease model. J. Appl. Microbiol. https://doi.org/10.1111/jam.14773 (2020).

43. Davis, D. J. et al. Lactobacillus plantarum attenuates anxiety-related behavior and protects against stress-induced dysbiosis in adult zebrafish. Sci. Rep. 6, 33726 (2016).

44. Park, M. R. et al. Probiotic Lactobacillus fermentum strain JDFM216 stimulates the longevity and immune response of Caenorhabditis elegans through a nuclear hormone receptor. Sci. Rep. 8, 1-10 (2018).

45. Plant, L., Lam, C., Conway, P. L. \& O’Riordan, K. Gastrointestinal microbial community shifts observed following oral administration of a Lactobacillus fermentum strain to mice. FEMS Microbiol. Ecol. 43, 133-140 (2003).

46. Wang, G. et al. The ameliorative effect of a Lactobacillus strain with good adhesion ability against dextran sulfate sodium-induced murine colitis. Food Funct. 10, 397-409 (2019).

47. Dinan, T. G. \& Cryan, J. F. Microbes, immunity, and behavior: psychoneuroimmunology meets the microbiome. Neuropsychopharmacology 42, 178-192 (2017).

48. Jang, S. Y. et al. Genome characteristics of Lactobacillus fermentum strain JDFM216 for application as probiotic bacteria. J. Microbiol. Biotechnol. 27, 1266-1271 (2017).

49. Romo-Araiza, A. et al. Probiotics and prebiotics as a therapeutic strategy to improve memory in a model of middle-aged rats. Front. Aging Neurosci. 10, 416 (2018).

50. Savignac, H. M., Kiely, B., Dinan, T. G. \& Cryan, J. F. Bifidobacteria exert strain-specific effects on stress-related behavior and physiology in BALB/c mice. Neurogastroenterol. Motil. 26, 1615-1627 (2014).

51. Slyepchenko, A., Carvalho, A. F., Cha, D. S., Kasper, S. \& McIntyre, R. S. Gut emotions-mechanisms of action of probiotics as novel therapeutic targets for depression and anxiety disorders. CNS Neurol. Disord. Drug Targets 13, 1770-1786 (2014).

52. Wang, H., Lee, I.-S., Braun, C. \& Enck, P. Effect of probiotics on central nervous system functions in animals and humans: a systematic review. J. Neurogastroenterol. Motil. 22, 589-605 (2016).

53. Cleal, M. et al. The Free-movement pattern Y-maze: a cross-species measure of working memory and executive function. Behav. Res. Methods https://doi.org/10.3758/s13428-020-01452-x (2020).

54. Maldonado Galdeano, C., Cazorla, S. I., Lemme Dumit, J. M., Vélez, E. \& Perdigón, G. Beneficial effects of probiotic consumption on the immune system. Ann. Nutr. Metab. 74, 115-124 (2019).

55. Yan, F. \& Polk, D. B. Probiotics and immune health. Curr. Opin. Gastroenterol. 27, 496-501 (2011).

56. de Moreno de Le Blanc, A. et al. Effect of the administration of a fermented milk containing Lactobacillus casei DN-114001 on intestinal microbiota and gut associated immune cells of nursing mice and after weaning until immune maturity. BMC Immunol. 9, 27 (2008).

57. Galdeano, C. M. \& Perdigón, G. Role of viability of probiotic strains in their persistence in the gut and in mucosal immune stimulation. J. Appl. Microbiol. 97, 673-681 (2004).

58. Yau, J. L. W. et al. Intrahippocampal glucocorticoids generated by $11 \beta-H S D 1$ affect memory in aged mice. Neurobiol. Aging 36, 334-343 (2015).

59. Hor, Y.-Y. et al. Lactobacillus strains alleviated aging symptoms and aging-induced metabolic disorders in aged rats. J. Med. Food 22, 1-13 (2019).

60. Lew, L.-C. et al. Lactobacillus strains alleviated hyperlipidemia and liver steatosis in aging rats via activation of AMPK. Int. J. Mol. Sci. 21, $5872(2020)$.

61. Fabian, E., Majchrzak, D., Dieminger, B., Meyer, E. \& Elmadfa, I. Influence of probiotic and conventional yoghurt on the status of vitamins B1, B2 and B6 in young healthy women. Ann. Nutr. Metab. 52, 29-36 (2008).

62. Baeeri, M. et al. a-Lipoic acid prevents senescence, cell cycle arrest, and inflammatory cues in fibroblasts by inhibiting oxidative stress. Pharmacol. Res. 141, 214-223 (2019).

63. Molz, P. \& Schröder, N. Potential therapeutic effects of lipoic acid on memory deficits related to aging and neurodegeneration. Front. Pharmacol. 8, 849 (2017).

64. Gebara, E., Udry, F., Sultan, S. \& Toni, N. Taurine increases hippocampal neurogenesis in aging mice. Stem Cell Res. 14, 369-379 (2015).

65. Fontana, L. \& Klein, S. Aging, adiposity, and calorie restriction. JAMA 297, 986-994 (2007).

66. Kannan, K. \& Jain, S. K. Effect of vitamin B6 on oxygen radicals, mitochondrial membrane potential, and lipid peroxidation in $\mathrm{H}_{2} \mathrm{O}_{2}$-treated U937 monocytes. Free Radic. Biol. Med. 36, 423-428 (2004).

67. Xiong, S., Patrushev, N., Forouzandeh, F., Hilenski, L. \& Alexander, R. W. PGC-1a modulates telomere function and DNA damage in protecting against aging-related chronic diseases. Cell Rep. 12, 1391-1399 (2015).

68. Sun, Q., Jia, N., Yang, J. \& Chen, G. Nrf2 signaling pathway mediates the antioxidative effects of taurine against corticosteroneinduced cell death in Human SK-N-SH cells. Neurochem. Res. 43, 276-286 (2018).

69. Xu, H. et al. Protective effect of Lactobacillus rhamnosus GG and its supernatant against myocardial dysfunction in obese mice exposed to intermittent hypoxia is associated with the activation of Nrf2 pathway. Int. J. Biol. Sci. 15, 2471-2483 (2019). 
70. Wang, Y. et al. Bacillus amyloliquefaciens SC06 protects mice against high-fat diet-induced obesity and liver injury via regulating host metabolism and gut microbiota. Front. Microbiol. 10, 1161 (2019).

71. Kim, D. H. et al. The seed extract of Cassia obtusifolia ameliorates learning and memory impairments induced by scopolamine or transient cerebral hypoperfusion in mice. J. Pharmacol. Sci. 105, 82-93 (2007).

72. Shiotsuki, H. et al. A rotarod test for evaluation of motor skill learning. J. Neurosci. Methods 189, 180-185 (2010).

73. Muller, K. A., Ryals, J. M., Feldman, E. L. \& Wright, D. E. Abnormal muscle spindle innervation and large-fiber neuropathy in diabetic mice. Diabetes 57, 1693-1701 (2008).

74. Marin, I. A. et al. Microbiota alteration is associated with the development of stress-induced despair behavior. Sci. Rep. 7, 43859 (2017).

75. Biller-Takahashi, J. D., Takahashi, L. S., Saita, M. V., Gimbo, R. Y. \& Urbinati, E. C. Leukocytes respiratory burst activity as indicator of innate immunity of pacu Piaractus mesopotamicus. Braz. J. Biol. 73, 425-429 (2013).

76. Kozich, J. J., Westcott, S. L., Baxter, N. T., Highlander, S. K. \& Schloss, P. D. Development of a dual-index sequencing strategy and curation pipeline for analyzing amplicon sequence data on the MiSeq Illumina sequencing platform. Appl. Environ. Microbiol. 79, 5112-5120 (2013).

77. Schloss, P. D. et al. Introducing mothur: open-Source, Platform-independent, community-supported software for describing and comparing microbial communities. Appl. Environ. Microbiol. 75, 7537-7541 (2009).

78. Quast, C. et al. The SILVA ribosomal RNA gene database project: improved data processing and web-based tools. Nucleic Acids Res. 41, D590-D596 (2013).

79. Cole, J. R. et al. Ribosomal database project: data and tools for high throughput rRNA analysis. Nucleic Acids Res. 42, D633-D642 (2014).

80. Westcott, S. L. \& Schloss, P. D. OptiClust, an improved method for assigning amplicon-based sequence data to operational taxonomic units. $m$ Sphere 2, e00073-17 (2017).

81. Rognes, T., Flouri, T., Nichols, B., Quince, C. \& Mahé, F. VSEARCH: a versatile open source tool for metagenomics. PeerJ 4, e2584 (2016).

82. Kanehisa, M., Sato, Y., Kawashima, M., Furumichi, M. \& Tanabe, M. KEGG as a reference resource for gene and protein annotation. Nucleic Acids Res. 44(D1), D457-D462 (2016).

83. Kanehisa, M. \& Goto, S. KEGG: Kyoto encyclopedia of genes and genomes. Nucleic Acids Res. 28, 27-30 (2000).

\section{Acknowledgements}

This research was supported by National Research Foundation of Korea (NRF) funded by the Ministry of Science, Information and Communications Technology (ICT) and Future Planning (2016M3C1B5907057) and Basic Science Research Program through the NRF funded by the Ministry of Education (2016R1A6A1A03012862).

\section{Author contributions}

M.R.P., M.S., and D.M. carried out the all experiments, and participated in drafting the manuscript. G.K. and T.U. performed the microbial community analysis. S.Y.J., D.Y.J., M.S., Y.K., and S.O. participated in all experimental design and coordination and helped to draft the manuscript. All authors read and approved the final manuscript.

\section{Competing interests}

The authors declare no competing interests.

\section{Additional information}

Supplementary information is available for this paper at https://doi.org/10.1038/s41598-020-77587-w.

Correspondence and requests for materials should be addressed to Y.K. or S.O.

Reprints and permissions information is available at www.nature.com/reprints.

Publisher's note Springer Nature remains neutral with regard to jurisdictional claims in published maps and institutional affiliations.

(c) (i) Open Access This article is licensed under a Creative Commons Attribution 4.0 International cc) License, which permits use, sharing, adaptation, distribution and reproduction in any medium or format, as long as you give appropriate credit to the original author(s) and the source, provide a link to the Creative Commons licence, and indicate if changes were made. The images or other third party material in this article are included in the article's Creative Commons licence, unless indicated otherwise in a credit line to the material. If material is not included in the article's Creative Commons licence and your intended use is not permitted by statutory regulation or exceeds the permitted use, you will need to obtain permission directly from the copyright holder. To view a copy of this licence, visit http://creativecommons.org/licenses/by/4.0/.

(C) The Author(s) 2020 